Www.jmscr.igmpublication.org Impact Factor 5.244

Index Copernicus Value: 83.27

ISSN (e)-2347-176x ISSN (p) 2455-0450

crossref DOI: https://dx.doi.org/10.18535/jmscr/v4i12.131

Journal Of Medical Science And Clinical Research

\title{
To study common cause of UTI in Female patients coming to Hospital with Special focus to restrain Antibiotic resistance
}

\author{
Authors
}

\author{
Dr Mohini Nainani ${ }^{1}$, Dr Manish Pokra ${ }^{2}$, Dr Yogendra Kumar Tiwari ${ }^{3}$ \\ ${ }^{1}$ Assistant Professor, Department of Obs-Gyn,Jhalawar Medical College \\ ${ }^{2}$ PG Resident, Department of Microbiology, Jhalawar Medical College \\ ${ }^{3}$ Assistant Professor, Department of Microbiology, Jhalawar Medical College
}

\begin{abstract}
ABTRACT
Urinary tract infections (UTIs) are conditions frequently complained by women both in the general population and in the hospital setting. Indeed it has been estimated that one woman out of three will experience at least an episode of UTI during lifetime. Urinary tract infections (UTIS) are one of the most frequent clinical bacterial infections in women, accounting for nearly 25\% of all infections. Around 50-60\% of women will develop UTIs in their lifetimes. Resistance in Gram-negative bacteria has been increasing, particularly over the last 6 years. This is mainly due to the spread of strains producing extended-spectrum $\beta$-lactamases (ESBLs) such as CTX-M enzymes or AmpC $\beta$ lactamases. Many of the isolates producing these enzymes are also resistant to trimethoprim, quinolones and aminoglycosides, often due to plasmid co-expression of other resistance mechanisms. CTX-M-producing Escherichia coli often occurs in the community and as E. coli is one of the commonest organisms causing urinary tract infections (UTIS) the choice of agents to treat these infections is diminishing. This study aimed to find bacterial profile of urinary tract infection (UTI) in female patients. This is a hospital based retrospective study conducted at Government Medical College, Jhalawar over a period of September 2015 to November 2016. Clean catch mid-stream urine samples from 500 clinically suspected cases of urinary tract infections were processed as per standard procedures. Among the 500 patients, 88 were found to be culture positive.
\end{abstract}

\section{Introduction}

Urinary tract infections (UTIs) occur more often in women than in men, at a ratio of $8: 1$. Approximately $50-60 \%$ of women report at least one UTI in their lifetime, and one in three will have at least one symptomatic UTI necessitating antibiotic treatment by age $24^{\cdot(-\underline{3})}$ Urinary Tract Infection (UTI) implies the presence of actively multiplying organisms in the urinary tract ${ }^{(4,5)}$ and it occurs in $3-5 \%$ of girls and $1 \%$ of boys during childhood, while it is more common in boys during the first year of life ${ }^{(6)}$. Every year about 250 million people worldwide are affected by UTIs. UTI is the third most common cause of admission to hospitals in India. It has been estimated that about 6 million patients per year are visited worldwide for UTI, out of which around 30,000 are treated in the wards ${ }^{(7)}$. Urinary Tract Infection (UTI) is among the most common serious bacterial infections in infants and children, and is frequently missed, probably because of its non-specific presentation ${ }^{(8)}$. It distresses the child and concerns the parents. It has a wide variety of clinical presentation, ranging from the asymptomatic presence of bacteria in the urine to potentially life-threatening infection of the kidney (9). Although, UTI is mainly due to ascending infection from the urethra, ${ }^{(10)}$ micro-organisms may reach the urinary tract by hematogenous or lymphatic routes as well. But ascending route 
accounts for almost $95 \%$ of cases of UTI ${ }^{(11)}$. Common nonbacterial causes of UTI include hemorrhagic cystitis from adenovirus and Candida infection in immunocompromised individuals. Common bacterial pathogens include gramnegative species such as Escherichia coli, Klebsiella, Proteus, Enterobacter, Pseudomonas, and Serratia spp. and gram-positive organisms, including group B streptococci, Enterococcus sp., and Staphylococcus aureus ${ }^{(12)}$. Escherichia coli is the most frequent bacteria to cause UTI in infants and children. The diagnosis of UTI remains complicated by nonspecific symptoms and the difficulty in obtaining an uncontaminated urine specimen. The most recent guidelines issued by the American Academy of Pediatrics (1999) suggest that a UTI should be considered in infants and children who present with symptoms and they should also be evaluated with a urinalysis and urine culture ${ }^{(13)}$.

In recent years, widespread use of antibiotics has been resulted in an increasing incidence of antibiotic resistance among the urinary tract pathogens all over the world. Worldwide, emerging of antibiotic resistance is increasing among the urinary pathogens ${ }^{(14,15)}$. The increase in resistance of microorganisms to antimicrobial agents, especially in hospitalized patients, demands rapid identification of the pathogens $(16,17,18)$. The knowledge of etiology and antibiotic sensitivity and resistance pattern of the organisms causing urinary tract infection is essential ${ }^{(19)}$. The aim of this study was to isolation and identification of uropathogens causing urinary tract infection and to obtain data on resistant pattern of these pathogens. Normally, the urinary tract is sterile, but bacteria may rise from the perianal region, possibly leading to UTI. Pathogens in the bladder may stay silent or can cause irritative symptoms like urinary frequency and urgency, and $8 \%$ of women may have asymptomatic bacteriuria.

If bacteria enter the blood stream, they could cause severe complications, including septicaemia, shock and, rarely, death. ${ }^{(20,21)}$ The definition of recurrent urinary tract infection (RUTI) is three UTIs with three positive urine cultures during a 12-month period, or two infections during the previous 6 months. ${ }^{(21-24)}$ UTIs are classified into 6 categories. The first category is an uncomplicated infection; this is when the urinary tract is normal, both structurally and physiologically, and there is no associated disorder that impairs the host defense mechanisms. The second category is an complicated infection; this is when infection occurs within an abnormal urinary tract, such as when there is ureteric obstruction, renal calculi, or vesicoureteric reflux. The third category, an isolated infection, is when it is the first episode of UTI, or the episodes are 6 months apart. Isolated infections affect $25-40 \%$ of young females. The fourth category, an unresolved infection, is when therapy fails because of bacterial resistance or due to infection by two different bacteria with equally limited susceptibilities. The fifth category, reinfection, occurs where there has been no growth after a treated infection, but then the same organism regrows two weeks after therapy, or when a different microorganism grows during any period of time. ${ }^{(25,26)}$ This accounts for $95 \%$ of RUTIs in women. Bacterial persistence happens when therapy is impaired by the accumulation of bacteria in a location that cannot be reached by antibiotics, such as infected stones, urethral diverticula and infected paraurethral glands. The sixth category, relapse, is when the same microorganism causes a UTI within two weeks of therapy; however, it is usually difficult to distinguish a reinfection from a relapse. ${ }^{(27)}$

\section{Materials and Methods}

To identify a UTI, keep an eye out for the following symptoms:

- A burning feeling when you urinate

- A frequent or intense urge to urinate, even though little comes out when you do

- Pain or pressure in your back or lower abdomen

- Cloudy, dark, bloody, or strange-smelling urine 
- Feeling tired or shaky

- Fever or chills (a sign the infection may have reached your kidneys)

Urine samples collected from suspected patients on the basis of above symptoms were sent to Microbiology laboratory within 6 hours of collecting samples. The samples were inoculated on blood agar and mac conkey agar and incubated at $37 \mathrm{oC}$ for 24 hours. All the clinical isolates were examined morphologically for colony characteristics on agar media. Those exhibiting mucoid colonies were processed for biochemical testing. Biochemical test employed were urease production, citrate utilization and fermentation of sugars. Sugar fermentation tests performed were sucrose, glucose, mannitol, lactose, adonitol, dulcitol, melibiose and esculin. Indole test and $\mathrm{H} 2 \mathrm{~S}$ production on TSI agar, oxidase, catalase and nitrate were also carried out. Besides these tests, motility and growth of organism in potassium cyanide were also checked. For biochemical tests standard procedures were used ${ }^{[28]}$. Antibiotic sensitivity testing was done for all the isolates on Mueller-hinton agar/Nutrient agar by modified Kirby-bauer disc diffusion technique. Antibiotic used were azithromycin (AZM), gentamicin (GM), augmentin (AUG),ceftriaxone (CTR), tobramycin (TOB), ceftazidime (OR), cefixime (CFM), piperacillin-tazobactam (PIT), imipenem (IMP), meropenem (MRO), chloramphanicol (C), ciprofloxacin (CIP), ofloxacin (OF), amikacin (AK), gentamycin (HLG), doxycycline(DO), cefoxitin (CX), norfloxacin (NX), nitrofurantoin (NIT), netilmicin (NIT), cotrimoxazole(COT).

\section{Result and Conclusion}

With emerging knowledge on antibiotic resistance and health care-associated infection, guidelines need to be updated to reflect the need to prescribe narrow spectrum agents when available and avoid empirical use of broad spectrum antibiotics. The most common organism causing the urinary tract infection in this study was Escherichia coli and Enterococcus spp. ,. Other were Klebsiella spp. ,
Proteus spp., Pseudomonas spp. , Coagulase negative staphylococcus, Citrobacter spp., Acinetobacter spp., and Staphylococcus aureus . E. coli is most sensitive to Ceftriaxone-sulbactum followed by nitrofurantoin and cotrimoxazole respectively. Enterococcus spp. is most sensitive to cotrimoxazole and norfloxacin.. In our studies we have found that Klebsiella spp. from clinical cases were highly susceptible to Netilimicin, Tobramycin, Azithromycin, Amikacin, Gentamicin Norfloxacin, Nitrofurantoin, Ofloxacin comparatively. Studies also shows that antibiotic such as Augmentin, Ceftazidime, Cefixime are 100 $\%$ resistant and Meropenem and imipenem were $11.5 \%$ susceptible which is due to bacteriaproducing Klebsiella pneumoniae carbapenemases (KPCs). Klebsiella isolates were found to show resistance to cefotaxime, ceftazidime, cefepime, cefoxitin, ceftriaxone. Almost 10 isolates were $100 \%$ resistant to all antibiotics used which were like a superbug to those patients and eye opener for medical fraternity. Our study shows that aminoglycosides like netilimicin, tobramycin, gentamicin, amikacin are potent antibiotics to some extent with susceptibility of $36.5 \%, 34.6 \%, 21.1 \%, 30.7 \%$ which is also not satisfactory and it indicates that Klebsiella is getting resistant to them also.As per result of study Pseudomonas were most susceptible to aminoglycosides (Tobramycin-66\%, Netilimicin54\%, Gentamicin-54\%, Amikacin-45\%) followed by fluoro-quinolones (Norfloxacin - 49\%, Ciprofloxacin- 18\%) a $n$ d Carbapenems (Meropenem-24\%, Imipenem-21\%). Piperacillintazobactum was almost $24 \%$ susceptible and Doxycycline, Ceftriaxone, Nitrofurantoin, Azithromycin were among least susceptible. A strategy of observing patients for possible symptoms before initiating antibiotics have been advocated, although recognizing this may not always be practical in a primary care setting. Reducing inappropriate prescribing of antibiotics for asymptomatic bacteriuria is likely to reduce antimicrobial resistance. 
E.coli Enterococcus klebsiella $\square$ Proteus $\square$ Pseudomonas

CNS Citrobacter Staph. aureus

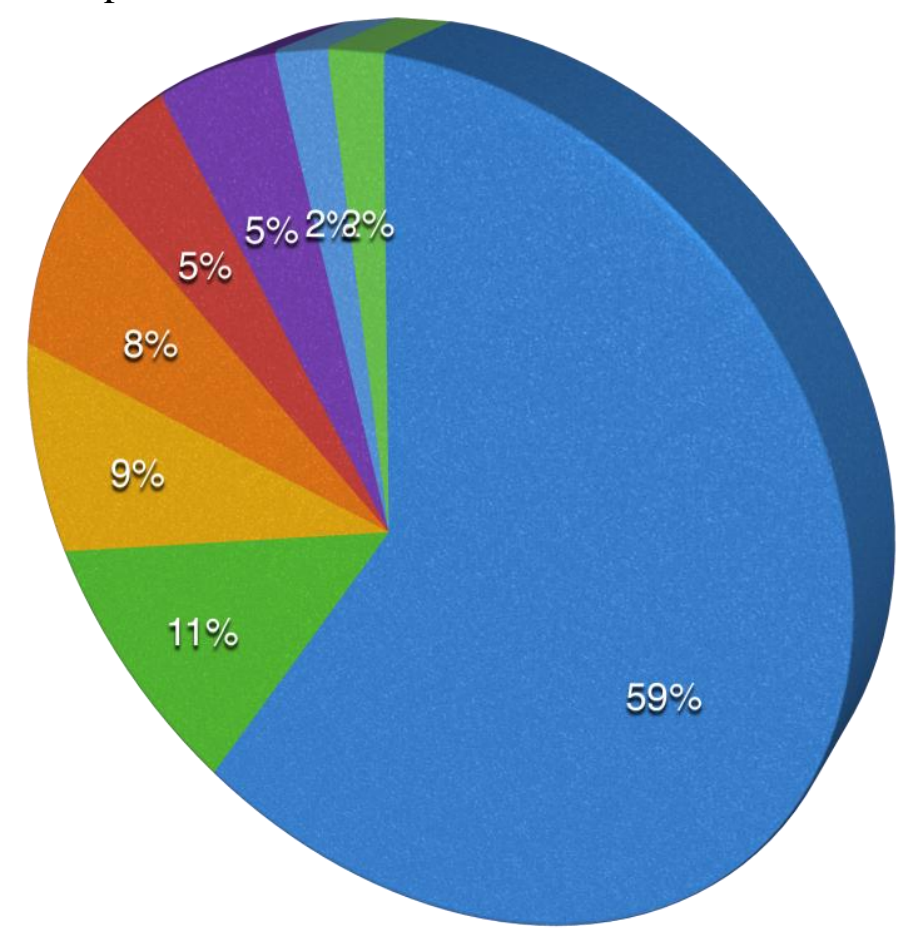

Figure.1 Distribution of Organisms Isolated from Urine Samples

Antibiotic sensitivity of top three causative agents-

\section{E.coli}

Ampi-sulbactum

Cephoxitin

\section{Cotri}

Linezolid
Nor

Cephalexin
Nitro Cipro $\square$ Levo

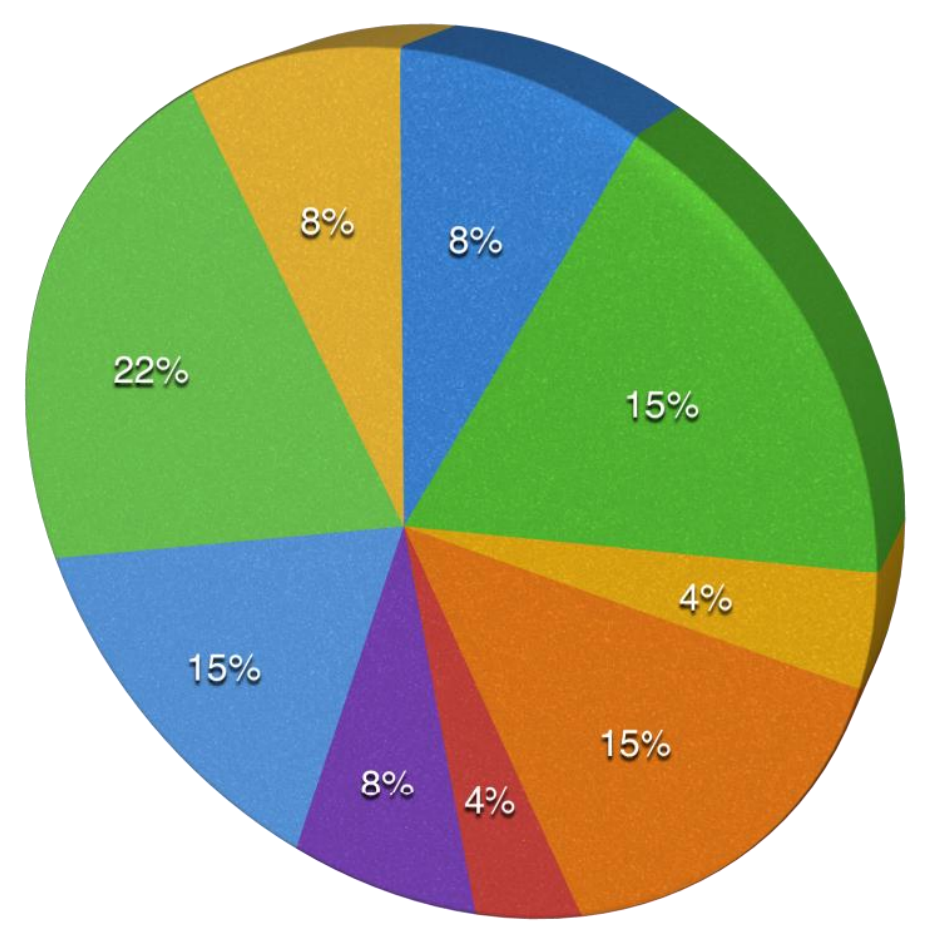




\section{Enterococcus}

Amp-sulbactum

Nitrofurantoin

Ceftriaxone

Norfloxacin
Ceft-sulbactum

Cipro cotrimoxazole

Levofloxacin

60

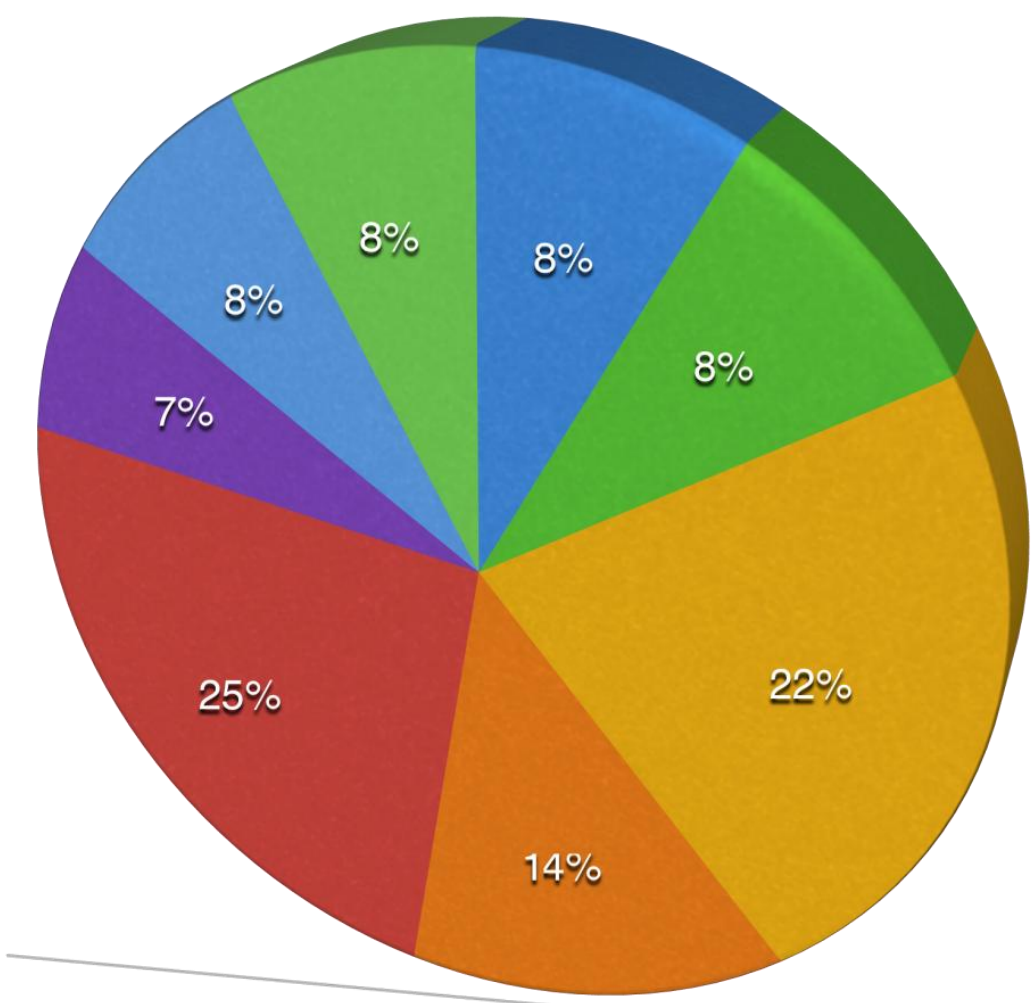

\section{Klebsiella}

Klebsiella

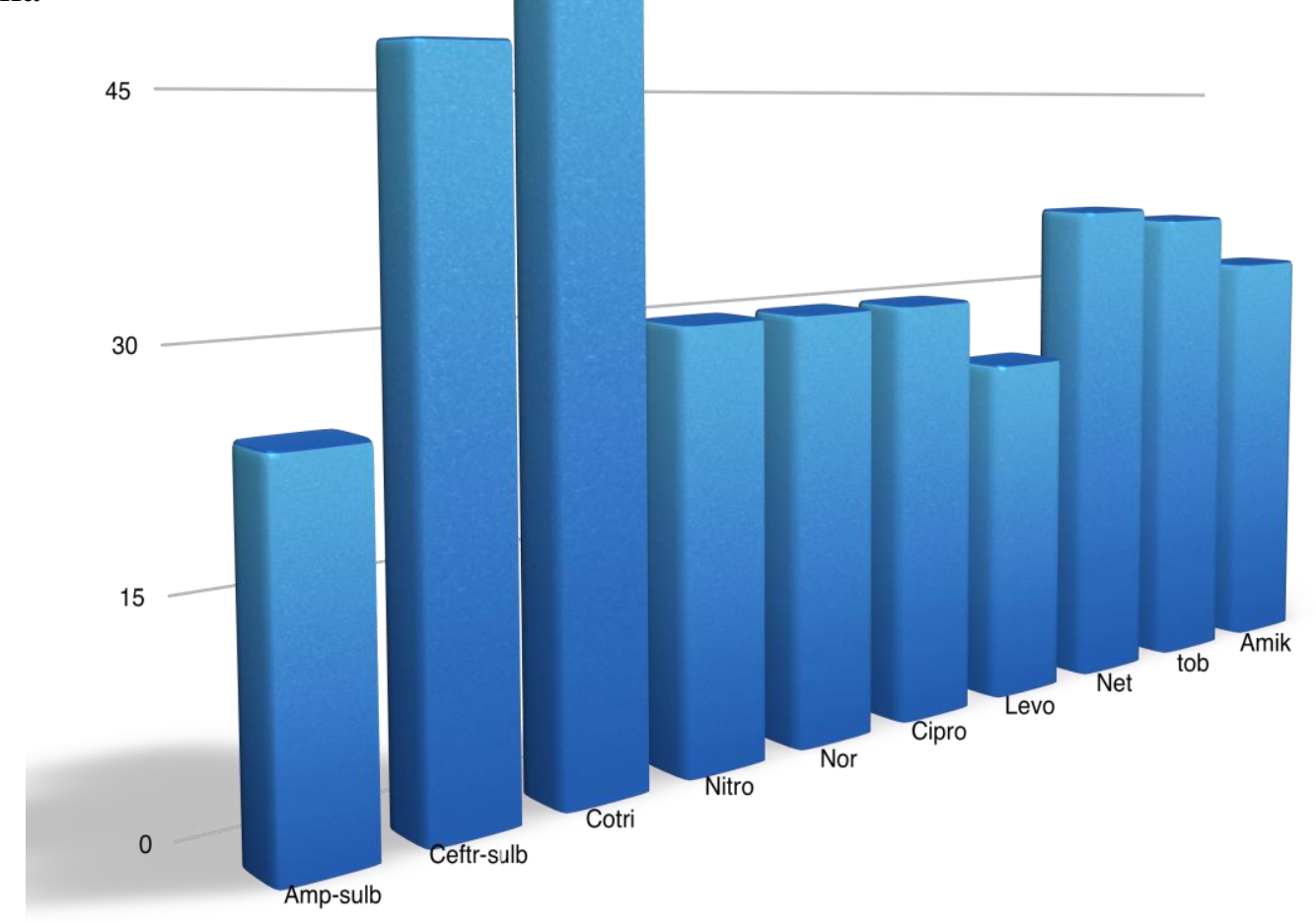




\section{Pseudomonas}

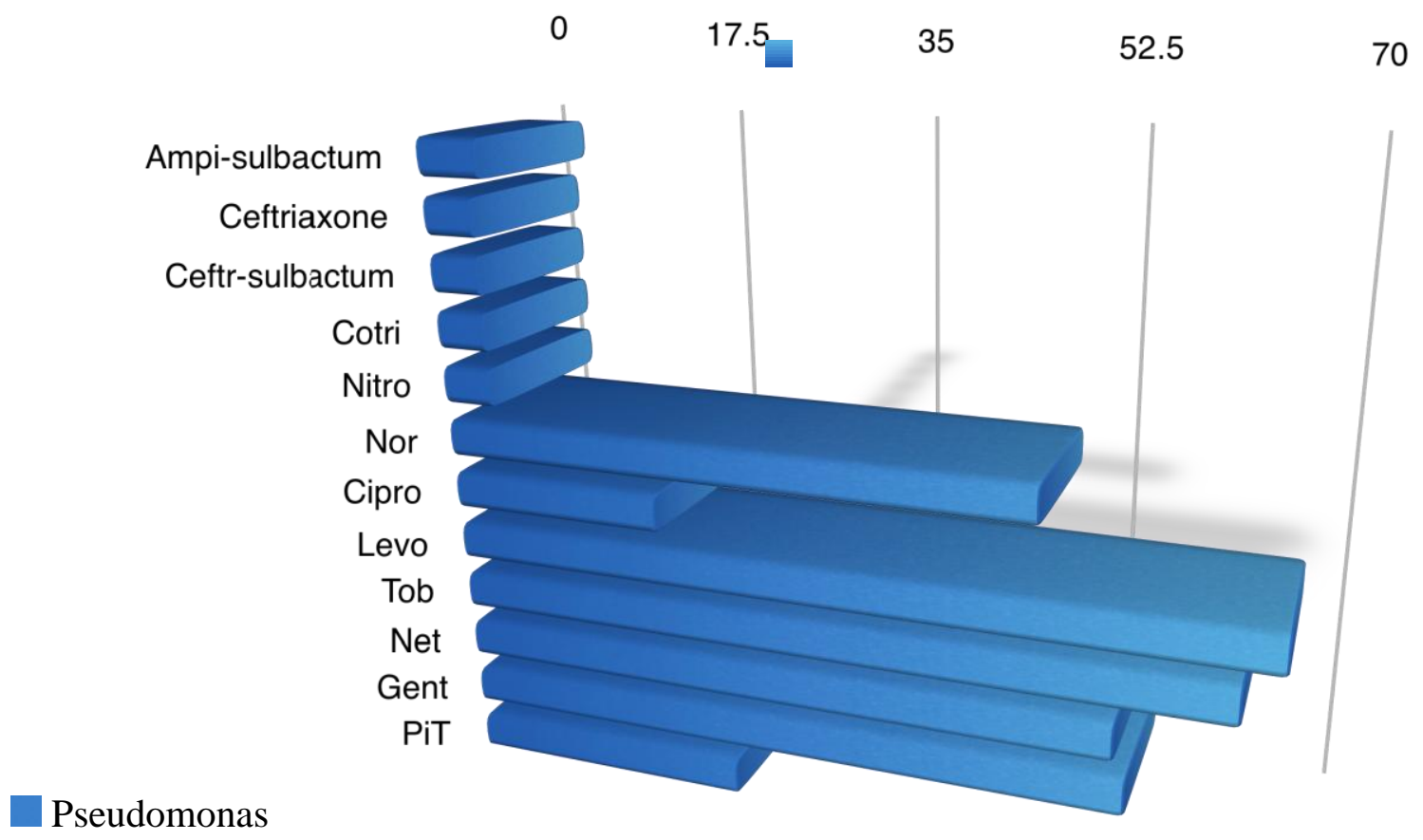

\section{Acknowledgement}

I have taken efforts in this project. However, it would not have been possible without the kind support and help of many individuals and organization. I would like to extend my sincere thanks to all of them. I am highly indebted to HODs of Department of Obs -Gyn and Department of Microbiology for their guidance and constant supervision as well as for providing necessary information regarding the project \& also for their support in completing the project. I would like to express my gratitude towards my parents \& member of medical college for their kind cooperation and encouragement which help me in completion of this project. I would like to express my special gratitude and thanks to Dr. Manish Pokra PG-2nd year resident, Dept. of Microbiology, for giving me time. My thanks and appreciations also go to my colleague in developing the project and people who have willingly helped me out with their abilities.

\section{Reference}

1. Rahn DD. Urinary tract infections: contemporary management. Urol Nurs. 2008;28:333-41. [PubMed]

2. Foxman B, Barlow R, D'Arcy H, Gillespie $\mathrm{B}$, Sobel JD. Urinary tract infection: selfreported incidence and associated costs. Ann Epidemiol. 2000;10:509-15. [PubMed]

3. Foxman B. Epidemiology of urinary tract infections: incidence, morbidity, and economic costs. Am J Med. 2002;113:511S. [PubMed]

4. Singh, S.D., Madhup, S.K. 2013. Clinical profile and antibiotics sensitivity in childhood urinary tract infection at Dhulikhel Hospital. Kathmandu Univ. Med. J. (KUMJ), 11(44): 319.

5. Lambert, H., Coulthard, M. 2003. The child with urinary tract infection. Clinical Paediatric Nephrol., 3: 197-225.

6. Owa, J.A. 1999. Urinary tract infections in children. In: Azubuike JC, Nkangineme K. E., editors. Paediatrics and Child Health in 
a Tropical Region. Owerri: African Educational services, pp. 480-1.

7. Bano, K., Khan, J., Begum, R.H., Munir, S., Akbar, N., Ansari, J.A., Anees, M. 2012. Patterns of antibiotic sensitivity of bacterial pathogens among urinary tract infections (UTI) patients in a Pakistani population. Afri. J. Microbiol. Res., 6(2): 414-20.

8. Akash, S., Adarsh, E. 2014. ClinicoMicrobiological Profile of UTI in Children less than 10 years in Department of Pediatrics in Tertiary Care Centre. J. Evol. Med. Dent. Sci., Vol. 3, Issue 15, April 14; Page: $\quad 3874-\quad 3882$, DOI: 10.14260/jemds/2014/2370.

9. Chang, S.L., Shortliffe, L.D. 2006. Pediatric urinary tract infections. Pediatric Clin. North America, 53(3): 379-400.

10. Sharma, A., Shrestha, S., Upadhyay, S., Rijal, P. 2011. Clinical and bacteriological profile of urinary tract infection in children at Nepal Medical College Teaching Hospital. Nepal Med Coll. J.,13(1): 24-26.

11. Goldman, M., Lahat, E., Strauss, S., Reisler, G., Livne, A., Gordin, L., Aladjem, M. 2000. Imaging after urinary tract infection in male neonates. Pediatrics, 105(6): 1232-1235.

12. Zorc, J.J., Kiddoo, D.A., Shaw, K.N. 2005. Diagnosis and management of pediatric urinary tract infections. Clin. Microbiol. Rev., 18(2): 417-422.

13. Johnson, J.R. 2003. Microbial virulence determinants and the pathogenesis of urinary tract infection. Infect. Dis. Clin. North America, 17(2): 261-278.

14. Kahlmeter, G. 2003. An international survey of the antimicrobial susceptibility of pathogens from uncomplicated urinary tract infections: the ECO. SENS Project. $J$. Antimicrob. Chemother, 51(1): 69-76.

15. Runehagen, R., Kahlmeter, G. 2002. A 10year study of the consumption of quinolones, trimethoprim and mecillinam in relation to the development of antimicrobial resistance in a large number of species. Clin. Microbiol. Infection, 8 (Supplement 1): 64.

16. Ashkenazi, S., Even-Tov, S.M.A.D.A.R., Samra, Z., Dinari, G. 1991. Uropathogens of various childhood populations and their antibiotic susceptibility. The Pediatric Infect. Dis. J., 10(10): 742-745.

17. Murray, B.E. 1989. Problems and mechanisms of antimicrobial resistance. Infect. Dis. Clin. North America, 3(3): 423-439.

18. Alon, U., Davidai, G., Berant, M., Merzbach, D. 1987. Five-year survey of changing patterns of susceptibility of bacterial uropathogens to trimethoprimsulfamethoxazole and other antimicrobial agents. Antimicrob. Agents and Chemother., 31(1): 126- 128.

19. Akash, S., Adarsh, E. 2014. ClinicoMicrobiological Profile of UTI in Children less than 10 years in Department of Pediatrics in Tertiary Care Centre. J. Evol. Med. Dent. Sci., Vol. 3, Issue 15, April 14; Page: $3874-\quad 3882$, DOI: 10.14260/jemds/2014/2370.

20. Mignini L, Carroli G, Abalos E, Widmer M, Amigot S, Nardin JM, et al. World Health Organization Asymptomatic Bacteriuria Trial Group Accuracy of diagnostic tests to detect asymptomatic bacteriuria during pregnancy. Obstet Gynecol. 2009;113:346-52.[PubMed]

21. Mohsin R, Siddiqui KM. Recurrent urinary tract infections in females. J Pak Med Assoc. 2010;60:55-9.[PubMed]

22. Albert X, Huertas I, Pereiro I, Sanfelix J, Gosalbes V, Perrotta C. Antibiotics for preventing recurrent urinary tract infection in non-pregnant women. Cochrane Database Syst Rev. 2004;3:CD001209. [PubMed]

23. Gopal M, Northington G, Arya L. Clinical symptoms predictive of recurrent urinary 
tract infections. Am J Obstet Gynecol. 2007;197:74.e1-4. [PubMed]

24. Foster RT., Sr Uncomplicated urinary tract infections in women. Obstet Gynecol Clin North Am. 2008;35:235-48. [PubMed]

25. American College of Obstetricians and Gynecologists ACOG Practice Bulletin No. 91: Treatment of urinary tract infections in nonpregnant women. Obstet Gynecol. 2008;111:785-94. [PubMed]

26. Karram MM, Mallipeddi PK. Lower urinary tract infection. In: Walters MD, Karram MM, editors. Urogynecology \& Reconstructive Pelvic Surgery. 2nd ed. St Louis: Mosby; 1999. pp. 341-53.

27. O'Reilly M. Recurrent urinary tract infection. In: Stanton SL, Dwyer PL, editors. Urinary Tract Infection in the Female. London: Martin Dunitz; 2000. pp. 227-40.

28. Cruickshank.R. Medical Microbiology 1980, 12th eds. (revised reprint) Edinburg: Churchill Livingstone. 170 - 189. 